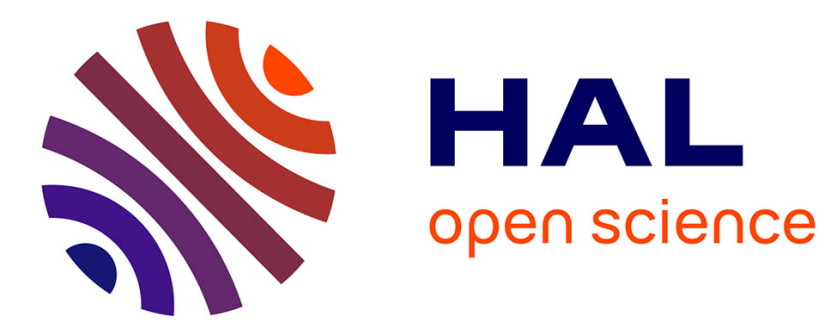

\title{
POWER-FLOW ANALYSIS USING INFINITE BEAM ELEMENTS
}

\author{
L. Gavric
}

\section{To cite this version:}

L. Gavric. POWER-FLOW ANALYSIS USING INFINITE BEAM ELEMENTS. Journal de Physique IV Proceedings, 1992, 02 (C1), pp.C1-511-C1-514. 10.1051/jp4:19921110 • jpa-00251065

\section{HAL Id: jpa-00251065 https://hal.science/jpa-00251065}

Submitted on 1 Jan 1992

HAL is a multi-disciplinary open access archive for the deposit and dissemination of scientific research documents, whether they are published or not. The documents may come from teaching and research institutions in France or abroad, or from public or private research centers.
L'archive ouverte pluridisciplinaire HAL, est destinée au dépôt et à la diffusion de documents scientifiques de niveau recherche, publiés ou non, émanant des établissements d'enseignement et de recherche français ou étrangers, des laboratoires publics ou privés. 


\title{
POWER-FLOW ANALYSIS USING INFINITE BEAM ELEMENTS
}

\author{
L. GAVRIC \\ CETIM, Département Acoustique Industrielle, BP. 67, F-60304 Senlis, France
}

\begin{abstract}
Numerical analysis of propagation phenomena in complex structures helps to predict their dynamical behavior. Attenuation and transmission paths of structure-borne sound can be analysed. The dynamic stiffness matrix of a semi-infinite beam, obtained by analytic methods can be used for such a purpose when the concerned substructure (structural part) is connected with the main structure by beams (wave-guides). In such a way, the whole structure has not to be modelled but only the structural part of interest. Both incident and reflected waves in wave-guides are taken into account by the method. The procedure can be applied to any computational technique having structural displacements as unknown quantities. An example is given to validate the approach.
\end{abstract}

\section{Introduction}

Propagation phenomena play an important role in dynamic behavior of large complex structures. The excitation force at one part of the structure induces waves which propagate the mechanical energy towards other structural parts and cause undesired vibrations and sound radiation far away from the excitation point. Very often, the energy is transfered by streight weakly damped beams of homogeneous cross-section (wave-guides). Although the mechanical energy propagates within such beams nearly without losses, the simple addition of a rigid body (blocking mass), causes practically the complete disappearance of all the wavelenghts shorter than a certain critical wavelenght from the transmitted flexural wave package. The simple formula obtained by the analytic solution of the wave equation can be used to find the inertial properties (mass and moment of inertia) of the rigid body needed to "block" certain wavelenghts. The analytic methods are not sufficient to predict the attenuation when the propagating wave is disrupted by a more complex substructure having distributed inertial, elastic and damping properties, but a numerical method has to be used. The method proposed use the conventional finite element procedures to evaluate elastic, inertial and damping properties of the substucture. Wave-guides are taken into account by the dynamic stiffness matrices of semi-infinite beams, which can absorbe the energy propagated within the finite element model. The mechanical energy entering the finite element model is introduced by the force vector, excerted to the structure by wave-guides, due to corresponding incident wave motions.

\section{Dynamic stiffness of semi-infinite beam}

The positively defined Cartesian co-ordinate system, used to evaluate the dynamic stiffness matrix of the semi-infinite beam has the origin in the point of attachement with the structure. The $x$-axis of the coordinate system, which coincides with the beam centerline, has the positive direction increasing out from the connection point along the semi-infinite part of the beam, while $y$ and $z$ axes coincide with the main axes of the cross-section of the beam. This co-ordinate system is reffered as local co-ordinate system of the semi-infinite beam. The displacement field for torsional and axial vibrations is supposed to consist of two waves. The first one propagates from the origin of the local coorinate system while the second propagates in the opposite direction, [1]. An exponentialy decaying near field component is added to the each of the two far field bending waves (bending in local $x-y$ and $x-z$ planes) in order to accomplish two boundary conditions (deflection and rotation) which can be imposed on each of the two flexural displacement fields at the attachement point, [1]. The Euler-Bernoulli equation for flexural vibrations is used. The forces excerted 
by the semi-infinite beam to the structure are then expressed by the displacements of the connection point by the following matrix equation:

$$
\left(\begin{array}{cccccc}
j \tilde{k}_{u} A \tilde{E} & 0 & 0 & 0 & 0 & 0 \\
0 & (j-1) \tilde{k}_{v}^{3} I_{z} \tilde{E} & 0 & 0 & 0 & j \tilde{k}_{v}^{2} I_{z} \tilde{E} \\
0 & 0 & (j-1) \tilde{k}_{w}^{3} I_{y} \tilde{E} & 0 & -j \tilde{k}_{w}^{2} I_{y} \tilde{E} & 0 \\
0 & 0 & 0 & j \tilde{k}_{\theta} J \tilde{G} & 0 & 0 \\
0 & 0 & -j \tilde{k}_{w}^{2} I_{y} \tilde{E} & 0 & (j+1) \tilde{k}_{w} I_{y} \tilde{E} & 0 \\
0 & j \tilde{k}_{v}^{2} I_{z} \tilde{E} & 0 & 0 & 0 & (j+1) \tilde{k}_{v} I_{z} \tilde{E}
\end{array}\right)\left(\begin{array}{c}
\tilde{u} \\
\tilde{v} \\
\tilde{w} \\
\tilde{\theta}_{x} \\
\tilde{\theta}_{y} \\
\tilde{\theta}_{z}
\end{array}\right)=\left(\begin{array}{c}
\tilde{N} \\
\tilde{Q}_{y} \\
\tilde{Q} \tilde{Q}_{z} \\
\tilde{T} \\
\tilde{M}_{y} \\
\tilde{M}_{z}
\end{array}\right)
$$

where $\tilde{u}, \tilde{v}$ and $\tilde{w}$ are the displacements parallel with local $x, y$ and $z$ axis. $\tilde{\theta}_{x}, \tilde{\theta}_{y}$ and $\tilde{\theta}_{z}$ are the rotations about the local $x, y$ and $z$ axis. $\tilde{N}$ is the axial force and $\tilde{T}$ is the moment of torsion. $\tilde{Q}_{y}$ and $\tilde{Q}_{z}$ are the shear forces in local $y$ and $z$ directions. $\tilde{M}_{y}$ and $\tilde{M}_{z}$ are the bending moments about local $y$ and $z$ directions. $\tilde{k}_{w}=\left(\omega^{2} \rho A / \tilde{E} I_{y}\right)^{1 / 4}$ and $\tilde{k}_{v}=\left(\omega^{2} \rho A / \tilde{E} I_{z}\right)^{1 / 4}$ are the wave numbers for bending in $x-z$ and $x-y$ planes, $\tilde{k}_{u}=\omega(\rho / \tilde{E})^{1 / 2}$ is the wave number for axial propagation and $\tilde{k}_{\theta}=\omega(\rho / \tilde{G})^{1 / 2}$ for torsional vibrations. Second moments of cross section are denoted $I_{y}$ and $I_{z}$, torsional moment of cross section is $J$ while $\tilde{E}$ and $\tilde{G}$ are Young's and shear moduli. The excitation frequency is $\omega$. The complex quantities are denoted by "tilde" , while $j$ is the imaginary unit. The influence of an incident wave is acounted for by an additional force vector $\left\{\tilde{f}_{\infty}\right\}$. If the forces, displacements and dynamic stiffness matrix, all reffered to Eq.(1), are denoted by $\{\tilde{f}\},\{\tilde{x}\}$ and $[\tilde{k}]$ respectively, the governing matrix equation in a case of incident wave reads:

$$
[\tilde{k}]\{\tilde{x}\}=\{\tilde{f}\}+\left\{\tilde{f}_{\infty}\right\} \quad ; \quad\left\{\tilde{f}_{\infty}\right\}=-2\left(\begin{array}{c}
j \tilde{k}_{u} A E \tilde{u}_{\infty} \\
(1-j) \tilde{k}_{v}^{3} \tilde{E} I_{z} \tilde{v}_{\infty} \\
(1-j) \tilde{k}_{w}^{3} \tilde{E} I_{y} \tilde{w}_{\infty} \\
j k_{\theta} \tilde{G} J \tilde{\theta}_{x \infty} \\
(j-1) \tilde{k}_{w}^{2} \tilde{E} I_{y} \tilde{w}_{\infty} \\
-(1-j) \tilde{k}_{v}^{2} \tilde{E} I_{z} \tilde{v}_{\infty}
\end{array}\right)
$$

where $\tilde{u}_{\infty}, \tilde{v}_{\infty}, \tilde{w}_{\infty}$ and $\tilde{\theta}_{x \infty}$ are the amplitudes of the incident wave components.

\section{Infinite beams assembled with finite element model}

The dynamical properties of a structural part discretized by the finite element method are defined by its mass, damping and stiffness matrices reffered to the global co-ordinate system of the finite element assembly, here denoted $[M],[H]$, and $[C]$ respectively. The influence of semi-infinite beams is accounted for by adding the corresponding dynamic stiffness matrix to the equation of motion. The excitation induced by the incident wave is accounted for by the excitation force vector defined in Eq.(2). Before the assembling, dynamic stiffness matrices of infinite beams and incident wave excitation vectors have to be transformed to the global co-ordinate system of the assembled structure, which is performed using conventional transformation procedures. The transformed dynamic stiffness matrices $[T]^{T}[\tilde{k}][T]$ and the excitation force vectors induced by incident waves $[T]^{T}\left\{\tilde{f}_{\infty}\right\}$ then have to be assembled to the global dynamic stiffness matrix $\left[\tilde{K}_{\omega}\right]$ and the global excitation force vector $\left\{\tilde{F}_{\omega}\right\}$, which account for constraints and excitations due to the whole group of semi-infinite beams used in the analysis:

$$
\left[\tilde{K}_{\omega}\right]=\sum[T]^{T}[\tilde{k}][T] \quad ; \quad\left\{\tilde{F}_{\omega}\right\}=\sum[T]^{T}\left\{\tilde{f}_{\infty}\right\} \quad ; \quad[T]=\left(\begin{array}{cc}
{[\lambda]} & 0 \\
0 & {[\lambda]}
\end{array}\right)
$$

where $[\lambda]$ is a three by three matrix of direction cosines of angles formed between local and global axes. The final equation of motion reads:

$$
\left(-\omega^{2}[M]+j \omega[H]+[C]+\left[\tilde{K}_{\omega}\right]\right)\{\tilde{X}\}=\left\{\tilde{F}_{\omega}\right\}
$$

where $\{\tilde{X}\}$ denotes displacement vector. Dynamic stiffnes matrix $\left[\tilde{K}_{\omega}\right]$ and excitation force vector $\left\{\tilde{F}_{\omega}\right\}$ have to be re-evaluated for each excitation frequency, which is emphasized by the subscript $\omega$. The matrix equation of motion is then solved frequency by frequency to obtain complex response of the structure $\{\tilde{X}\}$. From the known displacement field the net power injected or absorbed by the semi-infinite beam $P_{\text {in }}$ can be calculated using the following expression $(\Im(.$.$) denotes imaginary part):$

$$
P_{\text {in }}=-\frac{\omega}{2} \Im\left(\left\{\tilde{x}^{*}\right\}^{T}[\tilde{k}]\{\tilde{x}\}-\left\{\tilde{x}^{*}\right\}^{T}\left\{\tilde{f}_{\infty}\right\}\right)
$$


For the excitation frequencies comprised in the frequency range of the first few eigenfrequencies of the finite element model the equations of motion can be solved in modal co-ordinates, which diminishes the size of the problem and does not affect the accuracy of the computation of the input power and the power absorbed by the semi-infinite beams. On contrary, the spatial distribution of the energy transmitted within the structure is strongly affected by the limited number of modes used in the computation, which can be overcome using the solution of the corresponding static problem as proposed in [2] and [3].

\section{Structural intensity and net energy flow}

In assemblies of beams and plates various types of waves occure due to geometrical complexity of the structure, even if only one type of wave motion has been principally excited. The conversion between the flexural, torsional and longitudinal waves in beam assemblies and inplane and flexural waves in plates and shells, couples each of those effects and makes the analysis a very cumbersome task. Since, various wave types can have different amplitude units the simple distribution of lateral velocities may not be the best way to represent a propagation phenomenon consisting of different wave motions. The energy-related quantities have been imposed during the last decade to overcome this difficulty. Structural intensity and net energy flow (power-flux) are the two of them mostly used to describe the energy transfer in structures. The structural intensity $\{I\}$ is a vectorial quantity defined by the following relationship:

$$
\{I\}=-\frac{1}{2} \Re\left([\tilde{\sigma}]\left\{\tilde{v}^{*}\right\}\right)
$$

where $[\tilde{\sigma}]$ is tensor of stress component spectra and $\{\tilde{v}\}$ is vector, which contains spectra of particle velocity components. The "asterix" * denotes complex conjugate quantities and $\Re(.$.$) real part. The structural$ intensity equals the net energy flow through an infinitesimally small area. For the structural elements like beams, plates and shells which have one or two of their dimensions negligible compared with the others, it is convenient to integrate the structural intensity over them. The integration of structural intensity over the cross-section of a beam gives net energy flow in the beam. The obtained result is a vectorial quantity laying in the centerline of the beam. The integration of structural intensity over the thickness of a plate or a shell leads to the net energy flow per unith width of the plate or shell. Since the integration is performed in one dimension only, the integrated intensity remains a vectorial quantity laying in the plane tangential to the midsurface of the plate or the shell.

\section{Numerical computation of wave attenuation}

A numerical simulation of attenuation of incident wave is performed using a relatively modest finite element model and four semi-infinite beams. The structure consists of the rectangular plate $6[\mathrm{~mm}]$ thick, modelled by regular $6 \times 6$ finite element mesh, and two beams of a circular cross section of $20[\mathrm{~mm}]$ diameter, connected to the plate along the two sides, Fig.(1).
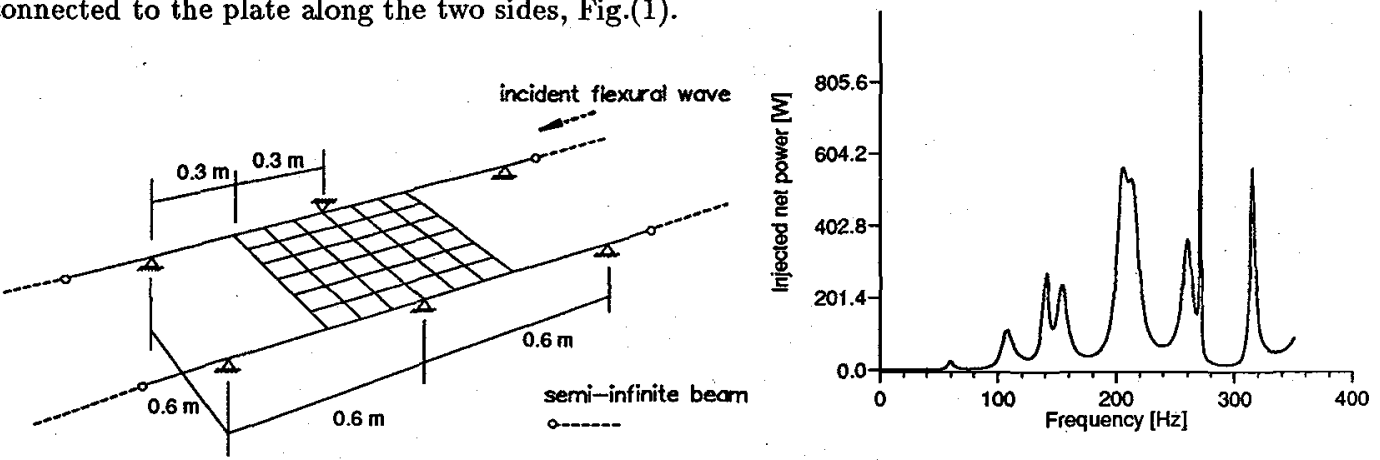

Fig. 1. Finite element model and net injected power by incident bending (flexural) wave

The beams are modelled by 16 finite elements each. The finite elements used are thin plate four node elements based on discrete Kirchhoff theory (DKQ) and beam type finite element based on Timoshenko's beam theory, [4], [5]. The four ends of the beams are connected with semi-infinite beams of the same geometry of the cross section, Fig.(1). The Young's modulus was taken 210000 [MPa], Poisson's ratio 
0.3 and mass density $7800\left[\mathrm{~kg} / \mathrm{m}^{3}\right]$, while material is considered dissipationless. The degrees of freedom corresponding to the inplane displacements of the plate-beam assembly are not taken into account. The finite element model has 188 degrees of freedom. The net power injected by the incident wave is computed for the frequency range $0-300[\mathrm{~Hz}]$ according $\mathrm{Eq} .(4)$. The incident wave is taken to be flexural with a displacement amplitude of $1[\mathrm{~mm}]$ for all frequencies considered. Since the structure is considered dissipationless the injected net power equals to the power transmitted through the substructure towards other structural parts. The transmitted net energy strongly depends on excitation frequency, Fig.(1). For some frequency ranges substructure strongly attenuates wave motion, while for the others the most of energy is transmitted through the substructure. The spatial distribution of the net energy propagated within the substructure is given in Fig. $(2-a, b, c)$ for the excitation frequencies of 45,90 and $135[\mathrm{~Hz}]$ respectively.

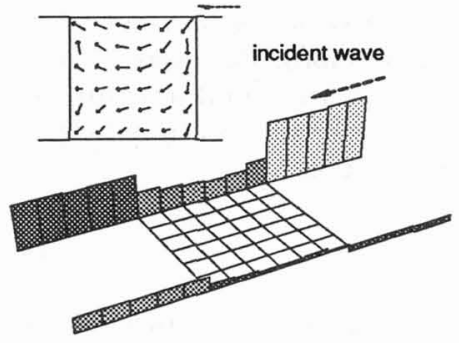

a)

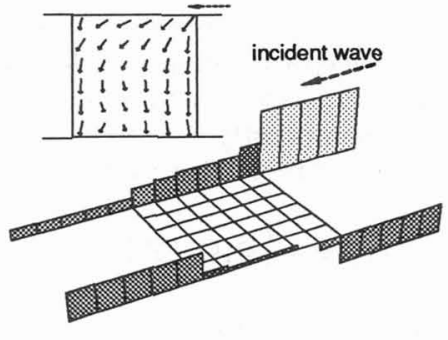

b)

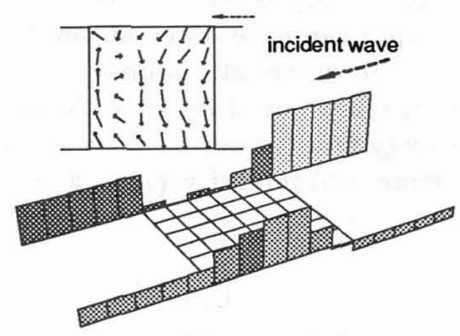

c)

Fig. 2. Net energy transmitted within plate and beams for three different excitation frequencies

The spatial distribution of the energy transmitted through the structure strongly depends on the excitation frequency. Some exctitation frequencies conserve the energy propagated in the incident wave beam (Fig.(2a) upper beam), some others transmit nearly all the energy through the plate to the initially non-excited beam (Fig.(2-b) lower beam). The net energy flow distribution in Fig.(2-c) shows an other interesting physical situation. The energy of the incident wave is transmitted towards the initially non-excited beam through the right part of plate, but most of the energy is then returned through the left part of the plate and absorbed by the upper beam. The energy flow distributions are evaluated by the integration of the structural intensity fields over the plate thickness and beam csoss-section.

\section{Conclusions}

A method is proposed for computation of propagation phenomena in substructures consisting of beams, plates and shells. The method uses standard finite element procedures to discretize the substructure. The dynamic stiffness matrices of semi-infinite beams are then included to the matrix equation of motion. They enable the transfer of the energy within the energetically "closed" mechanical system represented by the finite element model. The energy input (excitation) is provided by incident waves, which propagate towards the structure within semi-infinite beams. The method seems valuable in the domain of predictive computation.

\section{REFERENCES}

[1] - L. Cremer, M. Heckl: "Structure-borne sound" (translated and revised by E.E.Ungar) Springer-Verlag Berlin (1973).

[2] - L. Gavric: "Computation of structural intensity in thin-walled structures using FEM and modal approach", Proceedings of StruCoMe, Paris (1990).

[3]-L. Gavric, G. Pavic : "Computation of structural intensity in beam- plate structures by numerical modal analysis using FEM", Proceedings of $3^{e}$ Congrès international sur l'intensimetrie, Senlis (1990).

[4] - J. L. Batoz, G. Datt: "Modélisation des structures par éléments finis"Vol. 2 - Poutres et plaques, Hermès, Paris (1990).

[5] - J. S. Przemieniecky:"Theory of matrix structural analysis”, McGraw-Hill, (1982). 